\title{
Video Article \\ Kinematics and Ground Reaction Force Determination: A Demonstration Quantifying Locomotor Abilities of Young Adult, Middle-aged, and Geriatric Rats
}

\author{
Aubrey A. Webb ${ }^{1,2}$, Brendan Kerr ${ }^{3}$, Tanya Neville ${ }^{3}$, Sybil Ngan ${ }^{4}$, Hisham Assem ${ }^{3}$ \\ ${ }^{1}$ CullenWebb Animal Neurology \& Ophthalmology Center, Riverview, NB \\ ${ }^{2}$ Department of Clinical Neurosciences, Faculty of Medicine, University of Calgary \\ ${ }^{3}$ Department of Comparative Biology and Experimental Medicine, University of Calgary \\ ${ }^{4}$ Department of Neuroscience, University of Calgary
}

Correspondence to: Aubrey A. Webb at cullenwebb@me.com

URL: https://www.jove.com/video/2138

DOI: doi:10.3791/2138

Keywords: Neuroscience, Issue 48, Locomotion, kinetics, kinematics, aging

Date Published: 2/22/2011

Citation: Webb, A.A., Kerr, B., Neville, T., Ngan, S., Assem, H. Kinematics and Ground Reaction Force Determination: A Demonstration Quantifying Locomotor Abilities of Young Adult, Middle-aged, and Geriatric Rats. J. Vis. Exp. (48), e2138, doi:10.3791/2138 (2011).

\section{Abstract}

Behavior, in its broadest definition, can be defined as the motor manifestation of physiologic processes. As such, all behaviors manifest through the motor system. In the fields of neuroscience and orthopedics, locomotion is a commonly evaluated behavior for a variety of disease models. For example, locomotor recovery after traumatic injury to the nervous system is one of the most commonly evaluated behaviors ${ }^{1-3}$. Though locomotion can be evaluated using a variety of endpoint measurements (e.g. time taken to complete a locomotor task, etc), semiquantitative kinematic measures (e.g. ordinal rating scales (e.g. Basso Beattie and Bresnahan locomotor (BBB) rating scale, etc)) and surrogate measures of behaviour (e.g. muscle force, nerve conduction velocity, etc), only kinetics (force measurements) and kinematics (measurements of body segments in space) provide a detailed description of the strategy by which an animal is able to locomote ${ }^{1}$. Though not new, kinematic and kinetic measurements of locomoting rodents is now more readily accessible due to the availability of commercially available equipment designed for this purpose. Importantly, however, experimenters need to be very familiar with theory of biomechanical analyses and understand the benefits and limitations of these forms of analyses prior to embarking on what will become a relatively labor-intensive study. The present paper aims to describe a method for collecting kinematic and ground reaction force data using commercially available equipment. Details of equipment and apparatus set-up, pre-training of animals, inclusion and exclusion criteria of acceptable runs, and methods for collecting the data are described. We illustrate the utility of this behavioral analysis technique by describing the kinematics and kinetics of strain-matched young adult, middleaged, and geriatric rats.

\section{Video Link}

The video component of this article can be found at https://www.jove.com/video/2138/

Protocol

\section{Pre-requisites for Biomechanical Locomotion Analyses}

Prior to embarking upon purchasing expensive locomotor analysis equipment, and planning experiments where kinematics and/or kinetic locomotor assessment will be performed, it is imperative that the experimenter be familiar with the technical and practical aspects of biomechanical analyses, sensorimotor behavior, operant conditioning of animals, and handling/storing/managing large amounts of digitized data. Though these pre-requisites seem obvious to many, it is only after embarking upon these types of experiments where trainees realize the technical and practical complexities of performing relatively detailed locomotor analysis. The authors recommend that experimenters enroll in a course dealing with introductory biomechanics, be familiar with or hire someone familiar with a programming language required for data management, and of equal importance, spend substantial time interacting, handling, and working with laboratory animals. For understanding locomotion analysis and sensorimotor behavioral analysis in the neurosciences, experimenters are referred to several important references (see $1,4-7$ ).

\section{Kinematic and Kinetic Testing Apparatus}

A kinematic and kinetic testing apparatus, useful for collecting bilateral data, is comprised of the following components (see "Table of Specific Reagents and Equipment" for more detail):

- Quiet and sufficiently-sized room (not necessarily sound-proofed, though located in a low-traffic area) 
- Radio to supply background "noise" to limit distracting noises from outside the room to animals

- Clear plexiglass, enclosed, flat-surface runway $(180 \mathrm{~cm}$ long $X 20 \mathrm{~cm}$ wide $X 60 \mathrm{~cm}$ high) with a square hole $(11 \mathrm{~cm} X 11 \mathrm{~cm})$ located within the centre of the runway equidistant from either end

- Piece of plexi-glass $(10.5 \mathrm{~cm} \times 10.5 \mathrm{~cm})$

- Double-sided adhesive scotch tape

- $3 \mathrm{M}$ reflective adhesive tape for skin markers

- Hole punch for creating skin markers

- Isofluorane anesthesia machine required for anesthetizing animals for shaving prior to placement of skin markers

- Cheerios for encouraging movement of the animal along the length of the runway

- AMTI HE6X6 forceplate

- Vicon Motus customized system (software, computer, etc)

- 4 video cameras (at least $60 \mathrm{~Hz}$ sampling) and tripods with appropriate connector cables

- 4 camera-mounted halogen lights provides optimal lighting for illumination of the animal subject and the reflective skin markers

- Appropriate sized calibration frame or volume necessary for Vicon Motus system to calibrate $\mathrm{x}, \mathrm{y}, \mathrm{z}$ positions into meaningful distance measures

\section{Animal Training}

Prior to collecting data, each animal must be trained to cross a flat-surface, enclosed runway. Upon receiving rats from an appropriate animal supplier, animals should be acclimated to their new home for 1 week. During this acclimation time, several cheerios are placed daily into the rat's cage. Animals are food restricted to their maintenance energy requirements to prevent obesity and ensure motivation to perform this task. Thereafter, each animal is handled by the experimenter for 10-15 minutes daily for 1 week. During this same time period, each animal is placed into the runway with cheerios located at either end. Once the animal becomes familiar with their environment, they will begin eating the cheerios. Once the animal is comfortable and eating cheerios within the runway, the experimenter must then operantly condition the animal to run the length of the runway for a food reward. This is accomplished by tossing $1 / 4$ cheerio to the opposite end of the runway where the rat is positioned. Once the rat eats this cheerio, another $1 / 4$ cheerio is placed at the other end of the runway. This is done for $15-20$ minutes daily until the rat consistently (>90\% of tosses) moves along the runway at a constant velocity (i.e. without starting, stopping, exploring, or without changing gait) to eat the cheerio without galloping/bounding. The rat should only be employing a trotting gait. Over-conditioning of the animals to this task can lead to animals galloping and bounding these gaits are indicative of animals traveling $>90 \mathrm{~cm} / \mathrm{s}$. Bounding and galloping gaits, biomechanically, are more difficult to interpret for a variety of reasons (e.g. leg lead inclusion criteria, etc). In our experience, once rats consistently employ galloping or bounding gaits, it is difficult, if not impossible to have them use a trotting gait while locomoting in the runway. Velocities $>90 \mathrm{~cm} / \mathrm{s}$ are rarely seen after an animal has suffered from peripheral or central nervous system injury. Time to reach successful training is variable between strains and sexes of rats. Wistar, Lewis, Long-Evans, and Sprague-Dawley strains are able to consistently traverse the runway within 2 weeks from the onset of training ${ }^{8}$. In our experience, Fischer (F-344) rats tend to take upwards of 4-6 weeks to learn this task ${ }^{8}$.

\section{Joint Position Marking}

Forelimb kinematic analysis is unreliable due to skin movement artifact imposed by placing skin markers on the forelimbs which is exacerbated in species, like rats, that have a crouched posture 9 . Instead, kinematics of the forelimbs must be achieved using $x$-ray cinematography or fluoroscopy ${ }^{10-13}$. As such, hind limb joint position marker placement is only described herein.

Prior to data collection, each rat must be anesthetized at least 24 hours in advance of data collection using an appropriate inhalational anesthetic (e.g. isofluorane, $1.5-2 \%$ dialed on a precision vaporizor) and administered in oxygen via face mask, and key topographic anatomical landmarks must be marked. Given the brevity of the procedure, and because long-acting anesthetic agents are not used, use of an animal warming device need not be used to maintain the animal's body temperature. Once the animal is anesthetized, the hind limbs and the dorsum, to the level of the iliac crests are shaven. The animal is then placed in sternal recumbency and its hindlimbs are placed in an approximate standing position using firm packing foam to support it. The skin overlying the cranial-most portions of the iliac crests, the greater trochanter of the femur, the lateral tibial tuberosity, the tarsal joint, and the distal and lateral aspect of the $5^{\text {th }}$ metatarsal is marked with a non-toxic permanent marker. The animal is recovered from anesthesia. For temporal studies, periodic anesthesia may be required to shave the hindlimbs thereby permitting subsequent reflective marker placement (see below). Also, daily highlighting of the previously marked anatomical landmarks (using the same non-toxic marker) will be required as rats will slowly remove the markers through natural grooming behavior.

\section{Data Recording}

All camera views are examined to ensure that their position is appropriate and capturing the same field of view. Each camera should be placed at approximately 60-80 degrees to each other. The field of view should include the forceplate in the centre and a length of runway sufficient to capture two strides.

The calibration volume is placed within the pre-determined area of the runway. A single frame of the calibration volume within the runway, from each of the cameras, is captured. All calibrated marks along the length of each of the poles are digitized. Only once a satisfactory error in digitizing is accomplished, can the experimenter proceed to collecting locomotor data. This calibration step is critical prior to collecting data. If calibration is not performed accurately, or if calibration does not occur immediately prior to a recording session, all resulting data will be inaccurate and unusable. Importantly, if any of the cameras are touched or moved, it is safest to assume that calibration of the system needs to be repeated.

Immediately prior to placing the animal in the runway, its weight is recorded and pre-made conical reflective skin markers (using $3 \mathrm{M}$ reflective tape, see table) are adhered to the pre-determined felt marks made on the hind limb topographical landmarks. Recording the animal's weight will permit retrospective normalization of ground reaction forces to body weight - an important aspect if one wishes to make comparisons 
between groups. Additionally, body weight measurement facilitates monitoring of the animal's overall health for the duration of the experiment. Marker placement only requires appropriate animal handling and does not require anesthesia of the animal. If the adhesive on the reflective tape is insufficient to adhere to the animal's skin, a very small amount of non-toxic glue (e.g. 3M VetBond Tissue Adhesive) can be used to facilitate adherence of the marker on the animal's body. Once the markers are placed on the hindlimbs, the experimenter should be positioned comfortably near the keyboard of the computer and have in-hand the event marker attached through the Vicon Motus system. Using the calibrated file as a template, several files are made in advance of recording. Typically, 25 to 30 files need to be saved. Each file should be named uniquely. Each file will represent one recorded run of the animal being recorded. Twenty-five to 30 files are required to ensure that sufficient numbers of runs meeting the inclusion criterion velocity (i.e. $60-90 \mathrm{~cm} / \mathrm{s}$ ) are collected. After sufficient numbers of files are created the experimenter can begin collecting data. The experimenter must prompt the rat to shuttle within the runway by throwing $1 / 4$ pieces of cheerios at either end of the runway. With careful coordination and timing, the event marker is triggered at initiation and just prior to completion of the rat successfully completing a pass along the runway. After examining the crude ground reaction force tracing, and after recording whether the left or the right limbs hit the force plate, the file is saved and closed. Equal numbers of left and right limb forceplate hits should be recorded. The process of recording the data from a given run is repeated until sufficient numbers of runs have been recorded.

\section{Data Analysis}

Upon completing data collection for kinematics and ground reaction forces, each run from every animal needs to be evaluated for speed. Using two relatively fixed markers (e.g. wings of the iliums) one can evaluate a virtual point between the markers (done prior when creating a Vicon Motus file template). Before calculating the speed of this "virtual" point, markers for the iliac markers must be digitized. Using Vicon motus software, velocity of this virtual point in the X-direction (horizontal direction of movement) is calculated. In so doing, only runs within a given range speeds (determined a priori) are used in the final analyses. We find that animals moving between $60-90 \mathrm{~cm} / \mathrm{s}$ are using consistent trotting gaits. A minimum of 10 runs ( 5 runs where the left limb makes contact with the force platform and 5 runs where the right limb makes contact with the force platform) are required. Once the acceptable runs are identified for each animal, digitization of the remaining skin markers must be completed.

To compensate for skin movement artifact over the knee, estimation of the knee position is calculated using triangulation (intersection between two circles 2D kinematics; or intersection between 2 spheres 3D kinematics), as has been previously described. Hip, knee, and hock joint angles, velocities, and accelerations can now be determined. Stance and swing times can also be evaluated, though their accuracy is limited based upon the sampling speed of the cameras being employed. These and other calculations can be performed directly (i.e. without export) using Vicon Motus KineCalc software, or data can be exported as ASCII data and analyzed using customizable routines in software such as MatLab.

Ground reaction force data is measured and amplified by the AMTI force platform and collected at $1200 \mathrm{~Hz}$ by Vicon Motus. As such, once the ground reaction force data is collected, an appropriate digital filter is applied to the data using Vicon Motus. Given that the experimenter has already determined the speed of travel and identified acceptable runs after digitizing appropriate skin markers, ground reaction force data that was collected simultaneously as the kinematic data, can be analyzed using Vicon Motus KineCalc directly, or indirectly using some other customizable software routine. A variety of variables for forces, in each of the three orthogonal directions, can be calculated. Such variables include peak force, area und the curve (i.e. impulse), etc. Importantly, however, the experimenter must keep right and left limb data for each run of each animal separate. Data extracted from right or left limbs is averaged for each animal and used as the representative data for that animal Data is then analyzed using appropriate statistical procedures.

\section{Representative Results}

To represent the utility of this form of locomotor analysis, kinematics and ground reaction forces were determined for young, middle-aged, and geriatric female Wistar rats. From this analysis, age-related differences were found for female Wistar rats. In particular, ground reaction force analysis demonstrates that geriatric rats have reduced forelimb braking ability and tend to use their hindlimbs more for lateral stabilization compared to the other groups of animals (Figure 1). Kinematic analysis did not reveal any statistical differences between each group, though demonstrates that kinematics can be readily recorded from virtually any age of rat (Figure 2).
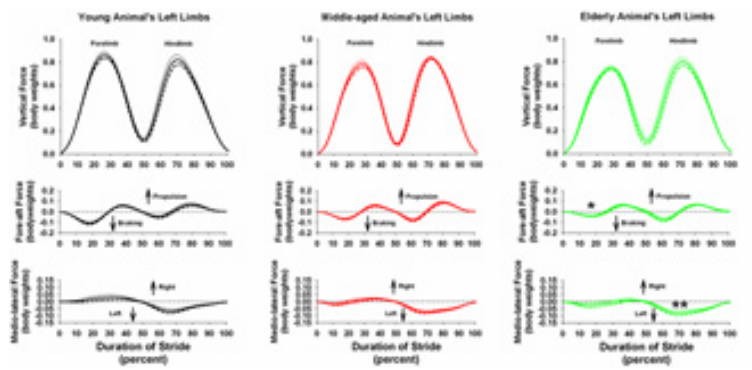

Figure 1. Ground reaction force tracing taken from the left limbs of young (4 month old; $n=7)$, middle-aged (13-14 months old; $n=7)$, and geriatric $(24$ months old; $n=5)$ female Wistar rats. Right limbs were similar. It is readily apparent that geriatric rats use their forelimbs less for braking ${ }^{*}=$ $\mathrm{p}<0.05)$ compared to young and middle-aged rats, and geriatric rats tend to use their hindlimbs more for lateral stabilization compared to young rats $\left(^{* *}\right)$. Solid lines represent mean, dotted lines represent mean + SE; dashed lines represent mean SE.Please click here to see a larger figure. 


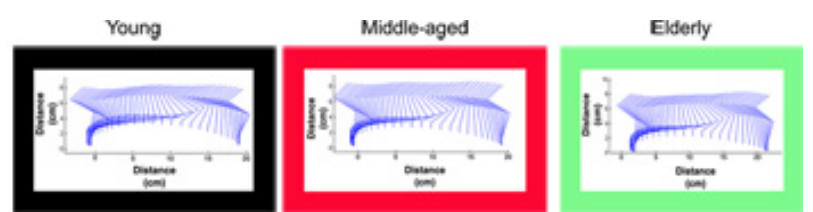

Figure 2. Kinematic stick figures for the left limb of young, middle-aged, and geriatric rats. Stick figures represent the mean movement of the left limb for each of the groups. There were no significant differences between the groups of animals for joint angle during the step cycle.Please click here to see a larger figure.

\section{Discussion}

The present paper provides methodology for evaluating locomotion using continuous quantitative kinematics and ground reaction force determination. Important for anyone interested in embarking upon this methodology is a strong background in biomechanics of locomotion, animal sensorimotor behavior, and data management and manipulation. Though kinematic and ground reaction force determination requires additional time and expertise, compared to some other forms of locomotor analysis (e.g. endpoint measures, ordinal rating scales), the data obtained is sensitive, objective and quantitative for a variety of orthopedic ${ }^{14-22}$ and neurologic ${ }^{1-3,23-32}$ models of disease, in a variety of species.

We have provided data that describes differences in locomotion between various ages of strain-matched rats information that could not be gleaned using simple and less sensitive measures. Further, kinematic and kinetic analysis of locomotion has been used to describe locomotor alterations in a variety of nervous system conditions where other forms of evaluation would be unsuccessful. ${ }^{8,23-25,27}$ The use of sensitive measures becomes especially important when evaluating potential therapeutants for various models of disease. If a test is not sensitive enough to discern an effect of a potential therapeutant the experimenter runs the risk of committing a type-II statistical error (i.e. concluding there is no effect of a treatment when in fact there was an effect). Further, because endpoint measures and more subjective tests that evaluate locomotion, there exists a potential for bias. Kinematic and kinetic evaluation is purely objective in that, provided appropriate inclusion/exclusion criterion are made a priori, the experimenter simply collects, examines and applies appropriate statistics to the data (i.e. there is no subjective component to data determination).

Kinematic and kinetic analysis also affords the ability to be used for a multitude of species. In fact, kinematics, ground reaction force determination, or both have been used in a variety of species such as elephants ${ }^{33-35}$, cattle $^{36}$, horses ${ }^{37-40}$, dogs ${ }^{4,41-45}$, cats ${ }^{21,46-49}$, various rodents $^{3,8,50,51}$, birds ${ }^{4,52-55}$, and fish ${ }^{56,57}$ (this list is by no means exhaustive). In the authors' experience, however, the use of mice is problematic given that mice are not easy to operantly condition to travel along a runway. Given this, mice will not travel at a relatively constant speed and instead speed-up and slow-down when traversing the runway. This behavior can likely, in part, be overcome by running mice on a treadmill and video-taping the animal locomoting on the treadmill. ${ }^{58}$ If the experimental apparatus for ground reaction force determination was to be modified for treadmill usage, ground reaction force determination would likely only be easily obtained for vertical ground reaction force as the treadmill belt would interfere with fore-aft and medio-lateral force determination.

Altogether, kinematic and kinetic analysis of locomotion is a reliable, sensitive, and objective method that can be employed for various models of orthopedic and neurologic conditions. Furthermore, all of the equipment has become available for use in rodents, thus negating any related reason for not performing this form of sensorimotor behavioral analysis.

\section{Disclosures}

No conflicts of interest declared.

\section{Acknowledgments}

This research was supported by a Discovery Grant to AAW from the Natural Sciences and Engineering Research Council of Canada

\section{References}

1. Muir, G.D. \& Webb, A.A. Mini-review: assessment of behavioral recovery following spinal cord injury in rats. Eur. J. Neurosci. 12, 3079-3086 (2000).

2. Costa, L.M. et al. Chapter 7: Methods and protocols in peripheral nerve regeneration experimental research: part IV-kinematic gait analysis to quantify peripheral nerve regeneration in the rat. Int. Rev. Neurobiol. 87, 127-139 (2009).

3. Webb, A.A. \& Muir, G.D. Sensorimotor behavior following incomplete cervical spinal cord injury in the rat. Behav. Brain Res. 165, 147-159 (2005).

4. Cavagna, G.A., Heglund, N.C., \& Taylor, C.R. Mechanical work in terrestrial locomotion: two basic mechanisms for minimizing energy expenditure. Am. J. Physiol 233, R243-R261 (1977).

5. Nigg, B.M., \& Herzog, W. (Eds.) Biomechanics of the Musculo-skeletal System. Wiley, New York, NY (2007).

6. Back, W., \& Clayton, H. (Eds.) Equine Locomotion. W.B. Saunders, (2000).

7. Alexander, R.M. Principles of Animal Locomotion. Princeton University Press, Princeton, NJ (2002).

8. Webb, A.A., Gowribai, K., \& Muir, G.D. Fischer (F-344) rats have different morphology, sensorimotor and locomotor abilities compared to Lewis, Long-Evans, Sprague-Dawley and Wistar rats. Behav. Brain Res. 144, 143-156 (2003).

9. Bauman, J.M. \& Chang, Y.H. High-speed X-ray video demonstrates significant skin movement errors with standard optical kinematics during rat locomotion. J. Neurosci. Methods 186, 18-24 (2010). 
10. Boczek-Funcke, A. et al. X-ray kinematic analysis of forelimb movements during target reaching and food taking in the cat. Eur. J. Neurosci. $12,1817-1826(2000)$.

11. Boczek-Funcke, A., Kuhtz-Buschbeck, J.P., \& Illert, M. X-ray kinematic analysis of shoulder movements during target reaching and food taking in the cat. Eur. J. Neurosci. 11, 986-996 (1999).

12. Boczek-Funcke, A. et al. X-ray kinematography as a tool for investigations of distal limb movements of the cat. J. Neurosci. Methods 52 , 11-16 (1994).

13. Alaverdashvili, M. et al. Cineradiographic (video X-ray) analysis of skilled reaching in a single pellet reaching task provides insight into relative contribution of body, head, oral, and forelimb movement in rats. Behav. Brain Res. 192, 232-247 (2008).

14. Sarver, J.J. et al. Transient decreases in forelimb gait and ground reaction forces following rotator cuff injury and repair in a rat model. J. Biomech. 43, 778-782 (2010).

15. van Klaveren, N.J. et al. Force plate analysis before and after dorsal decompression for treatment of degenerative lumbosacral stenosis in dogs. Vet. Surg. 34, 450-456 (2005).

16. Robinson, D.A. et al. The effect of tibial plateau angle on ground reaction forces 4-17 months after tibial plateau leveling osteotomy in Labrador Retrievers. Vet. Surg. 35, 294-299 (2006).

17. Conzemius, M.G. et al. Effect of surgical technique on limb function after surgery for rupture of the cranial cruciate ligament in dogs. J. Am. Vet. Med. Assoc. 226, 232-236 (2005).

18. Marsolais, G.S. et al. Kinematic analysis of the hind limb during swimming and walking in healthy dogs and dogs with surgically corrected cranial cruciate ligament rupture. J. Am. Vet. Med. Assoc. 222, 739-743 (2003).

19. Budsberg, S.C. et al. Force plate analyses before and after stabilization of canine stifles for cruciate injury. Am. J. Vet. Res. 49, 1522-1524 (1988).

20. Budsberg, S.C. et al. Prospective evaluation of ground reaction forces in dogs undergoing unilateral total hip replacement. Am. J. Vet. Res. 57, 1781-1785 (1996).

21. Romans, C.W. et al. Use of pressure platform gait analysis in cats with and without bilateral onychectomy. Am. J. Vet. Res. 65, 1276-1278 (2004).

22. Roush, J.K. et al. Evaluation of the effects of dietary supplementation with fish oil omega-3 fatty acids on weight bearing in dogs with osteoarthritis. J. Am. Vet. Med. Assoc. 236, 67-73 (2010).

23. Kemp S.W.P. et al. Behavioral and anatomical analysis of selective tibial nerve branch transfer to the deep peroneal nerve in the rat. Eur. J. Neurosci. 31, 1074-1090 (2010).

24. Muir, G.D. et al. Dorsolateral cervical spinal injury differentially affects forelimb and hindlimb action in rats. Eur. J. Neurosci. 25, 1501-1510 (2007).

25. Webb, A.A. \& Muir, G.D. Course of motor recovery following ventrolateral spinal cord injury in the rat. Behav. Brain Res. 155, 55-65 (2004).

26. Webb, A.A. \& Muir, G.D. Unilateral dorsal column and rubrospinal tract injuries affect overground locomotion in the unrestrained rat. Eur. J. Neurosci. 18, 412-422 (2003).

27. Webb, A.A. \& Muir, G.D. Compensatory locomotor adjustments of rats with cervical or thoracic spinal cord hemisections. J. Neurotrauma 19, 239-256 (2002).

28. Miklyaeva, E.I. et al. The ground reaction forces of postural adjustments during skilled reaching in unilateral dopamine-depleted hemiparkinson rats. Behav. Brain Res. 88, 143-152 (1997).

29. Muir, G.D. \& Whishaw, I.Q. Red nucleus lesions impair overground locomotion in rats: a kinetic analysis. Eur. J. Neurosci. 12, 1113-1122 (2000).

30. Muir, G.D. \& Whishaw, I.Q. Complete locomotor recovery following corticospinal tract lesions: measurement of ground reaction forces during overground locomotion in rats. Behav. Brain Res. 103, 45-53 (1999).

31. Muir, G.D. \& Whishaw, I.Q. Ground reaction forces in locomoting hemi-parkinsonian rats: a definitive test for impairments and compensations. Exp. Brain Res. 126, 307-314 (1999).

32. Muir, G.D. \& Steeves, J.D. Phasic cutaneous input facilitates locomotor recovery after incomplete spinal injury in the chick. J. Neurophysiol. 74, 358-368 (1995).

33. Genin, J.J. et al. Biomechanics of locomotion in Asian elephants. J. Exp. Biol. 213, 694-706 (2010).

34. Ren, L. et al. The movements of limb segments and joints during locomotion in African and Asian elephants. J. Exp. Biol. 211, 2735-2751 (2008).

35. Hutchinson, J.R. et al. The locomotor kinematics of Asian and African elephants: changes with speed and size. J. Exp. Biol. 209, 3812-3827 (2006).

36. van der Tol, P.P. et al. The vertical ground reaction force and the pressure distribution on the claws of dairy cows while walking on a flat substrate. J. Dairy Sci. 86, 2875-2883 (2003).

37. Nauwelaerts, S. \& Clayton, H.M. Trunk deformation in the trotting horse. Equine Vet. J. 41, 203-206 (2009).

38. Wickler, S.J. et al. Energetic and kinematic consequences of weighting the distal limb. Equine Vet. J. 36, 772-777 (2004)

39. Biknevicius, A.R., Mullineaux, D.R., \& Clayton, H.M. Ground reaction forces and limb function in tolting Icelandic horses. Equine Vet. J. 36 , 743-747 (2004).

40. Clayton, H.M. Instrumentation and techniques in locomotion and lameness. Vet. Clin. North Am. Equine Pract. 12, 337-350 (1996).

41. Walter, R.M. \& Carrier, D.R. Rapid acceleration in dogs: ground forces and body posture dynamics. J. Exp. Biol. 212, 1930-1939 (2009).

42. Lister, S.A. et al. Ground reaction force analysis of unilateral coxofemoral denervation for the treatment of canine hip dysplasia. Vet. Comp Orthop. Traumatol. 22, 137-141 (2009).

43. Williams, S.B. et al. Exploring the mechanical basis for acceleration: pelvic limb locomotor function during accelerations in racing greyhounds (Canis familiaris). J. Exp. Biol. 212, 550-565 (2009).

44. Gillette, R.L. \& Angle, T.C. Recent developments in canine locomotor analysis: a review. Vet. J. 178, 165-176 (2008).

45. Jayes, A.S. \& Alexander, R.M. Mechanics of locomotion of dogs (Canis familiaris) and sheep (Ovis aries). J. Zool. 185 Pt 3, $289-308$ (1978).

46. Frigon, A. et al. Asymmetric changes in cutaneous reflexes after a partial spinal lesion and retention following spinalization during locomotion in the cat. J. Neurophysiol. 102, 2667-2680 (2009).

47. Vilensky, J.A. \& Cook, J.A. Do quadrupeds require a change in trunk posture to walk backward? J. Biomech. 33, 911-916 (2000).

48. Belanger, M. et al. A comparison of treadmill locomotion in adult cats before and after spinal transection. J. Neurophysiol. 76, 471-491 (1996).

49. Belanger, M., Drew, T., \& Rossignol, S. Spinal locomotion: a comparison of the kinematics and the electromyographic activity in the same animal before and after spinalization. Acta Biol. Hung. 39, 151-154 (1988). 
50. Fischer, M.S. et al. Basic limb kinematics of small therian mammals. J. Exp. Biol. 205, 1315-1338 (2002)

51. Witte, H. et al. Torque patterns of the limbs of small therian mammals during locomotion on flat ground. J. Exp. Biol. 205, 1339-1353 (2002).

52. Muir, G.D. \& Gowri, K.S. Role of motor and visual experience during development of bipedal locomotion in chicks. J. Neurophysiol. 94, 3691-3697 (2005).

53. Muir, G.D. Locomotor plasticity after spinal injury in the chick. J. Neurotrauma 16, 705-711 (1999).

54. Reiter, K. \& Bessei, W. Gait analysis in laying hens and broilers with and without leg disorders. Equine Vet. J. Suppl110-112 (1997).

55. Griffin, T.M. \& Kram, R. Penguin waddling is not wasteful. Nature 408, 929 (2000).

56. Donley, J.M. et al. Convergent evolution in mechanical design of lamnid sharks and tunas. Nature 429, 61-65 (2004).

57. Katz, S.L., Syme, D.A., \& Shadwick, R.E. High-speed swimming. Enhanced power in yellowfin tuna. Nature 410, 770-771 (2001).

58. Leblond, H. et al. Treadmill locomotion in the intact and spinal mouse. J. Neurosci. 23, 11411-11419 (2003). 